\title{
Self-care and improved outcomes: an intervention by heart failure nurse specialists
}

Julie MacInnes, Principal Lecturer, Faculty of Health and Wellbeing, School of Nursing, Canterbury Christ Church University College, Canterbury; Anita Walton, Heart Failure Nurse Specialist, Eastern and Coastal Kent Community Health NHS Trust, Kent. Email: julie.macinnes@canterbury.ac.uk

\section{Abstract:}

Heart failure is a prevalent, debilitating condition. Patients need to develop and maintain effective strategies and behaviours to care for themselves and manage their illness.

Aim: The aim of this study was to evaluate whether or not an educational intervention carried out by a team of heart failure nurse specialists (HFNS), as part of usual care, enhanced self-care behaviour in patients with chronic heart failure.

Methods: A quantitative survey was carried out using the 'Looking After Yourself with Heart Failure Questionnaire (LAYHFQ)' to determine levels of self-care behaviour pre- and post-HFNS intervention. All new patients registered to a community heart failure nursing service completed the questionnaire at initial assessment and 6 weeks later following the nurse-led intervention $(N=36)$. Paired t-tests (2-tailed) were carried out to determine if there were significant differences between total self-care scores before and after the intervention, and for each individual self-care behaviour.

Results: Self-care was improved overall $(p=0.011)$ with five behaviours demonstrating a statistically significant improvement: namely the uptake of flu and pneumonia vaccination ( $p=0.009)$, regular weighing $(p=0.001)$, recognising changes in sleep patterns $(p=0.014)$, recognising signs of fluid retention $(p=0.001)$, and the wellbeing dimension of going out as much as possible $(p=0.019)$.

Conclusion: An educational intervention by an HFNS leads to enhanced self-care and may result in improved physical and psychological outcomes for patients with heart failure.

Key words:

Heart failure, Self-care, Nurse Specialist Intervention, Questionnaire Introduction:

Heart failure is a complicated progressive syndrome that occurs when the ability of the heart to pump enough blood around the body quickly enough is impaired, which results in the needs of the body not being met efficiently (National Institute for Health and Clinical Excellence (NICE), 2010; Sutherland, 2010). Heart failure can be caused by a number of disease processes that damage or overload the heart, such as myocardial infarction, hypertension and valvular heart disease. According to NICE (2010), 900000 people in the UK have heart failure and a further 60000 will develop the syndrome each year. Incidence and prevalence increase with age, with an average age at first diagnosis of 76 years. Prevalence is expected to rise significantly in the future owing to an ageing population, increased survival of people with coronary artery disease (CAD), and the improved and advancing efficacy of treatments for heart failure. This is anticipated to lead to further financial burden with existing costs estimated at $f 629$ million (Sutherland, 2010). This is in addition to the impact on the quality of health of individuals and their families and carers, which should not be underestimated (Iqbal et al, 2010). Patients with heart failure should be supported to develop and maintain effective strategies and behaviours to care for themselves and manage their illness (NICE, 2010). This is supported by UK government policy which emphasises self-care as a key principle in the management of long-term conditions ( $\mathrm{DH}, 2006)$. Strategies aimed at promoting selfcare are a routine intervention in the care of patients with heart failure in the community setting 
in which this study is based. The purpose of this study was to evaluate the effectiveness of heart failure nurse specialist (HFNS) intervention in enhancing the self-care of patients in this locality. Jaarsma et al (2003) define self-care as actions directed at the maintenance of a stable condition. Such actions include adherence to medication; refraining from behaviours that can worsen the condition such as drinking excess alcohol; and the ability to recognise early signs of decompensation, for example by monitoring daily weight and symptoms. Collaboration with health professionals is also an important aspect of self-care; for instance, seeking help when symptoms worsen.

A literature review was undertaken to identify key literature on the association between self-care in heart failure and outcomes, and the interventions that have been used to improve self-care in this population. The databases, CINAHL, Medline, PsychInfo and the British Nursing Index, were searched using the key words: heart failure, self-care, outcomes. These were used singularly and in combination. In addition, national and European guidelines for promoting self-care in heart failure were reviewed.

\section{Self-care and improved outcomes}

From this review, a number of European studies have identified reduced hospitalisation and use of emergency services linked to higher levels of self-care in heart failure. Lainscak et al (2011) for the European Society of Cardiology (ESC) identifies improved survival and fewer hospital admissions in patients who are more involved in their own care and treatment. Similarly, Jones et al (2012) found that adherence to weight-monitoring and self- adjustment of diuretic dose was linked to fewer emergency department attendances and admissions to hospital. Lee et al (2010) looked at all-cause mortality, admission and/or attendance at emergency departments as outcomes among patients who practised different levels of self-care and those that were symptom free. They found that the symptomatic heart failure patients who were above average in self-care, had an event-free survival benefit similar to that of symptom-free heart failure patients. They concluded that more effective self-care improves heart failurerelated morbidity. However, is should be noted that variables such as levels of social support and psychological factors including self-efficacy, are known to influence selfcare behaviours, and may therefore also influence outcomes (MacInnes, 2008).

Interventions to improve self-care

Educational interventions designed to improve self-care in heart failure have been described in European and International literature. Smeulders et al (2009) evaluated the effects of the Chronic Disease Self-Management Programme (CDSMP) on self-care. This was a randomised controlled trial with 317 patients who experienced limitations in activity, with follow-up for 1 year. They found that those undertaking the programme had significantly improved physical activity for up to 6 months following the intervention, compared with those receiving usual care. However, there was no impact on other health behaviour outcomes or improved health-care use. Shao et al (2013) conducted a randomised controlled trial with 47 patients in the intervention group. This group undertook a $12-$ week educational programme that focused on self-monitoring of salt and fluid intake and recognising heart failure-related symptoms. Participants who received the intervention programme had significantly better self-management behaviour, and self-efficacy for salt and fluid control, and their heart failure-related symptoms were significantly lower than participants in the control group. Baker et al (2011), in a large randomised controlled trial of 605 patients, found that a single, face-toface, education session, followed by up to 8 telephone sessions over 1 month, improved quality of life (as measured by an adapted Minnesota Living with Heart Failure Questionnaire, MLHFQ), health behaviours, and knowledge compared with a control group. Heydari et al (2011) examined the relationship between self-concept and adherence to medication in an Iranian heart failure 
population of 108 patients. They found that through counselling and education, nurses were in a position to empower patients to treat heart failure as a challenge rather than a threat, leading to greater self-reported adherence to prescribed drugs.

Guidelines: promotion of self-care

McMurray et al (2012), on behalf of the ESC, support a programme of holistic care aimed at reducing the risk of hospitalisation in heart failure patients. The promotion of self-care is recognised as an important component of this programme and consists of both education and skills training. Essential topics and skills should be taught in relation to self-care (McMurray et al, 2012). NICE (2010) discusses lifestyle advice along with rehabilitation in supporting patients with heart failure. The importance of discharge planning, and the need for instructions before leaving hospital on how to access help are also highlighted. The Scottish Intercollegiate Guidelines Network (SIGN) (2007) also gives comprehensive guidance on lifestyle advice and innovative models of care that can be used. According to Blue and Millerick (2011), for the management of heart failure to be truly comprehensive and optimal, it should include tailored education, as well as practical tips, advice and support for the carer(s). This should help to ensure the best quality of life, while at the same time, giving realistic expectations of care.

Methods and methodology:

Aim and design

The aim of this study was to evaluate whether or not the interventions carried out by a team of HFNS', as part of usual care, enhanced self-care behaviour in patients with chronic heart failure. A quantitative survey was carried out using a validated, structured questionnaire, the 'Looking After Yourself with Heart Failure Questionnaire' (LAYHFQ) (MacInnes, 2013), to determine levels of selfcare behaviour pre- and post-HFNS intervention. The intervention consisted of information, education, practical advice and support aimed at initiating and maintaining self-care strategies as part of a plan of care for all patients. The study was approved by the NHS Trust Research and Development Department. As this was an evaluation of usual care, rather than a primary research study, no further ethical approvals were necessary.

Participants

Data were collected over a period of 3 months during 2014 and included all new patients registered to the service over this time $(N=53)$. The service typically receives referrals from inpatient hospital admissions, cardiologist outpatient clinics, GPs, other specialist nurses and community matrons. The criteria for referral to the HFNS, and therefore, inclusion in the study, was a new diagnosis of left ventricular systolic dysfunction (LVSD) as confirmed by an echocardiogram. Patients were invited to complete the LAYHFQ as part of a usual assessment, which included demographic and clinical data such as age, gender, ejection fraction, baseline medication, observations, and aetiology of heart failure. Health-related quality of life data were also collected using the MLHFQ (Rector et al, 1987). This assessment was carried out by the HFNS at an initial home or clinic visit.

Data collection methods

Data were collected using the LAYHFQ (Maclnnes, 2013). This is a 26-item scale which assesses selfcare dimensions of lifestyle behaviour, medication adherence, symptom monitoring and management, and reporting and seeking help. Statements include 'I drink between 1.5-2 litres (2.53.5 pints) of fluid every day' and 'I weigh myself regularly'. Responses are scored using a 5-point Likert scale from 'strongly agree' to 'strongly disagree'. The questionnaire was self-completed by 
participants at the time of the initial clinical assessment and, again, 6 weeks later at a follow-up appointment. Internal reliability of this questionnaire is good ( $\alpha=0.77$ ) (MacInnes, 2013). Although other self-care questionnaires are available for use in heart failure, such as the Self-Care of Heart Failure Index (SCHFI) (Riegel et al, 2009), and the European Self-Care Behaviour Scale (EHFSCBS) (Jaarsma et al, 2003), the LAYHFQ has similar reliability and has been designed specifically for a UK heart failure population.

\section{The HFNS intervention}

This consisted of information, education and practical advice and support for initiating and maintaining self-care strategies as part of a plan of care for all patients. As part of the initial assessment, the HFNS provided a detailed explanation of the condition related to each individual patient's aetiology of heart failure. This was directly linked to the main symptoms of heart failure, specifically shortness of breath, oedema and fatigue. Self-care strategies were discussed which introduced the concept of daily weight as a means of monitoring fluid status. Typically, this was presented as the means by which the patient could take control of their condition, to some degree. Patients were asked to weigh on rising in morning, after visiting the toilet, but before getting dressed or eating and drinking. It was emphasised that a weight gain of $1.5 \mathrm{~kg}$ or more over two to three days should be highlighted to the HFNS or GP, particularly if accompanied by worsening symptoms. Information of how and when the heart failure service can be accessed, including the telephone helpline, was given to patients, as well as help in navigating NHS systems. Fluid intake of 2 litres daily was recommended with an increase of half a litre in exceptionally warm weather. Salt (sodium) intake was also discussed together with the avoidance of salt substitutes owing to the risk of interaction with medication. Healthy eating was also routinely discussed and supported by leaflets from the BHF. Strategies for medication management were discussed in detail, with explanations of the actions of drugs and the reasons for the need to optimise doses of beta-blockers and ACE inhibitors (ACEi). Advice regarding the annual flu injection and one-off pneumococcal vaccination was also given in order to minimise the risk of chest infections in the winter, and thus prevent symptom exacerbation. Over-the-counter medications were addressed, tailored to the individual, with warnings about St Johns Wort (owing to interactions with medications such as warfarin, digoxin and eplerenone), and the risks of taking non-steroidal anti-inflammatory drugs (NSAIDS). The importance of exercise in addition to achieving an appropriate amount of rest was emphasised. Heart failure is often characterised by 'good days and bad days', with the perceived need to achieve plenty of activity on a good day and then 'paying the price' on a bad day. Patients were encouraged to undertake an existing cardiac rehabilitation exercise programme, where appropriate. Smoking and alcohol consumption were routinely addressed with smoking cessation referrals offered where necessary. Abstinence from alcohol was advised when the cause of the heart failure is known to be alcoholic cardiomyopathy. In all other cases, the government daily guidelines of 2-3 units for women and 3-4 units for men were recommended.

Data analysis methods

Data was analysed using SPSS (v.20). Paired t-tests (2-tailed) were carried out to determine if there were significant differences between total self-care scores before and after the HFNS intervention and for each individual's self-care behaviour. P values of $<0.05$ were considered statistically significant. Results The number of patients who completed the initial questionnaire was 53 , which represents the total number of patients referred to the heart failure service over the 3-month period of the study. 35 patients completed the questionnaire at 6 weeks.

Results: 
Characteristics of the sample

Of the participants, $67 \%(n=24)$ were men and $33 \%(n=12)$ were women; The mean age was 73.5 years (range 48-94; SD \pm 11.86 ). The mean ejection fraction was $31 \%$ (range $20-55 \%$; SD \pm 7.36 ).

Total self-care scores

The total self-care score before the HFNS intervention was 26.07 (range -52 to $+52 ; \mathrm{SD} \pm 8.48$ ) and $31.24(S D \pm 9.19)$ after. This was a statistically significant improvement $(p=0.011)$. This is comparable to a previous study using this questionnaire where the baseline total self-care score was 24.86 (Maclnnes, 2013).

Individual self-care behaviour scores

Table 1 shows the mean scores for each self-care behaviour before and after the intervention and whether these differences were statistically significant.

\section{Discussion:}

Self-care, overall, was improved. When considering individual self-care behaviours, adherence to all lifestyle factors was reportedly increased. These included taking regular exercise, limiting fluid and salt intake, and restricting alcohol intake. However, these were not statistically significant. Improvements in these scores are likely to be a result of educational input, especially relating to those behaviours most specific to heart failure. A number of studies have documented that adherence to lifestyle behaviours are the most widely practised aspects of selfcare in heart failure (Smith et al, 2009). Adherence to medication also reportedly increased. The uptake of flu and pneumonia vaccination was statistically significant. Again, this is likely to be as a direct result of improved knowledge. The rationale for these vaccinations was given by the HFNS rather than issued as a simple instruction, which may be significant in improving adherence. Less unintentional nonadherence to medication was reported, with a reduced incidence of forgetting to take medication and making sure prescriptions were collected before running out. Similarly, intentional nonadherence was reduced, with fewer instances of patients choosing not to take their medication. The HFNS' reinforce the benefits of taking medication, which may have resulted in a stronger belief that the medication is necessary, leading to improved adherence. Beliefs about the necessity of medication have been shown to be a significant predictor of improved self-care in heart failure (Maclnnes, 2013). There was also a reported increase in changing the time of taking medication. This may be because the HFNS' were able to give 'permission' for patients to do this according to individual patient preferences and lifestyle. In terms of symptom monitoring, the incidence of regular weighing, recognising changes in sleep patterns and signs of fluid retention were all significantly improved. Weighing daily is often something that the patient and carer can do to help take control in what can be a frightening situation. It is a simple, practical and effective selfmanagement strategy. Jones et al (2012) cited this aspect of self-care as one of the main behaviours which contributed to fewer hospital admissions and linked to improved morbidity. The recognition of changes in sleeping pattern could also be linked to initial education about when to call for help in terms of worsening symptoms such as orthopnoea. A degree of anxiety may be present and the importance of responding to the reporting of this symptom should not be underestimated. Levels of anxiety and depression were not routinely assessed by the HFNS although referral can be made to a counselling service where deemed appropriate. Often, a need to discuss medication for depression and the use of counselling services can evolve as also high- lighted by Shao et al (2013). Recognising symptoms of fluid retention, such as worsening cough, ankle oedema and abdominal distension, as being related to fluid overload in heart failure is an important component of education, along with 
the importance of reporting these changes. Recognising and monitoring weight changes and other signs of overload can directly affect the need for admission to hospital (ESC, 2011). In this study, respiratory changes were least easily recognised; perhaps because these symptoms may already be perceived as severe. Importantly, the ability to recognise the effects of medication was improved. This may include diuresis or perceived side-effects such as light-headedness or nausea, for example. The difficulty in differentiating symptoms of heart failure from the effects of medication has been well documented in other studies (Rogers et al, 2002; Horowitz et al, 2004). Going out as much as possible was significantly improved. However, there was little change in reportedly maintaining a positive outlook and making an effort to see friends and family. These behaviours are an often overlooked aspect of self-care but relate closely to aspects of quality of life (Macabasco-O'Connell et al, 2011). Such complex, social phenomena are known to be influenced by a number of factors, including level of social support (Salyer et al, 2012), and psychological status such as levels of anxiety and depression (Makaya et al, 2013). Patients may lack the confidence to go out and continue activities after receiving a diagnosis of heart failure and well-meaning friends and family may advise 'taking it easy'. The importance of maintaining an active physical and social life, as much as possible, was emphasised by the HFNS. To facilitate this, patients were encouraged to inform their HFNS of any planned social events or other activities so that appointments could be scheduled around these. Patients reported being more likely to seek help and report changes in their health and the effects of medication. This is consistent with a greater awareness of symptoms and the effects of medication, and demonstrates a partnership approach to self-care, as envisaged by the $\mathrm{DH}$ (2006).

\section{Limitations:}

A number of limitations in this study are recognised. The multifaceted nature of the intervention by the HFNS makes it difficult to determine which aspects may have the greatest impact on influencing self-care. Although all the HFNS' were working to clinical protocols, there is likely to be some inconsistency in the way in which the intervention was delivered-especially as much of the information was necessarily tailored to individual patients. A number of factors are known to influence self-care such as social support, literacy levels and psychological factors such as anxiety and depression. These data were not routinely collected and so could not be correlated with selfcare in this study. This was a small sample size and a number of participants $(n=17)$ did not complete the questionnaire following the intervention. The main reason for this was that patients were discharged before completion of the second questionnaire $(n=12)$. Sadly, five patients died in this time period. Demographic data of those that were lost to follow-up were not analysed as part of this study. Results rely on accurate self-reporting of behaviours and there may have been a temptation to appear to be carrying out all of the expected behaviours - the so-called 'halo' effect. However, a study by Smith et al (2009) has shown similar levels of self-care using self-report, and seemingly more objective measures of behaviour such as observation, pill-counts and urinalysis for sodium levels.

\section{Conclusions and recommendations:}

The intervention by HFNS is effective in improving overall self-care in patients with heart failure with many individual self-care behaviours enhanced. It seems likely that the relationship built between the nurse and patient contributes to improved adherence to recommended lifestyle changes and presents a unique opportunity for nurses to deliver an individualised, patient-centred programme of care. Finally, there is an assumption that improved self-care results in improved patient outcomes, such as reduced hospital admissions, associated costs, and morbidity and mortality. Further research is needed to demonstrate links between improved self-care and these outcomes. Quality of life data 
were collected by the MLHFQ in this study and it would be interesting to compare these with the self-care data in a follow-up study.

Key Points:

- Patients with heart failure need to develop and maintain effective strategies and behaviours to care for themselves and manage their illness

- Self-care can be conceptualised as adherence to lifestyle behaviours and medication, monitoring and managing symptoms, promoting wellbeing, and seeking help and reporting changes

- The LAYHFQ is a validated instrument for measuring self-care in heart failure in a UK population

- An educational intervention by a heart failure nurse specialist can lead to enhanced self-care

- Further research is needed to demonstrate the relationship between self-care, clinical outcomes and quality of life

References:

Baker DW, Dewalt DA, Schillinger D et al (2011) The effect of progressive, reinforcing telephone education and counseling versus brief educational intervention on knowledge, self-care behaviors and heart failure symptoms. J Card Fail 17(10): 789-96.

Blue L, Millerick Y (2011) Nonpharmacological management. In: McDonagh TA, Gardner RA, Clark AL, Dargie H, eds. Oxford Textbook of Heart Failure. Oxford University Press, Oxford: 469-78

Department of Health (2006) Supporting people with long-term conditions to Self-Care: A guide to developing local strategies and good practice. DH, London. http://tinyurl.com/kd5zcnv (accessed 21 January 2016)

Heydari A, Ahrari S, Vaghee S (2011) The relationship between selfconcept and adherence to therapeutic regimens in patients with heart failure. J Cardiovasc Nurs 26(6): 475-80.

Leventhal H (2004) A story of maladies, misconceptions and mishaps: effective management of heart failure. Soc Sci Med 58(3): 631-43

Iqbal J, Francis L, Reid J, Murray S, Denvir M (2010) Quality of life in patients with chronic heart failure and their carers: a 3-year followup study assessing hospitalization and mortality. Eur J Heart Fail 12(9): 1002-8.

Jaarsma T, Strömberg A, Mårtensson J, Dracup K (2003) Development and testing of the European Heart Failure Self-Care Behaviour Scale. Eur J Heart Fail 5(3): 363-70

Jones CD, Holmes GM, Dewalt DA et al (2012) Is adherence to weight monitoring or weight-based diuretic self-adjustment associated with fewer heart failure-related emergency department visits or hospitalizations? J Card Fail 18(7): 576-84.

Lainscak M, Blue L, Clark AL et al (2011) Self-care management of heart failure: practical recommendations from the Patient Care Committee of the Heart Failure Association of the European Society of Cardiology. Eur J Heart Fail 13(2): 115-26.

Lee CS, Moser DK, Lennie TA, Riegel B (2011) Event-free survival in adults with heart failure who engage in self-care management. Heart Lung 40(1): 12-20. 
Macabasco-O'Connell A, DeWalt DA, Broucksou KA et al (2011) Relationship between literacy, knowledge, self-care behaviors, and heart failure-related quality of life among patients with heart failure. J Gen Intern Med 26(9): 979-86.

MacInnes J (2008) Factors affecting self-care in heart failure: A literature review. British Journal of Cardiac Nursing 3(7): 293-9.

Maclnnes J (2013) Relationships between illness representations, treatment beliefs and the performance of self-care in heart failure: a cross-sectional survey. Eur J Cardiovasc Nurs 12(6): 53643.

McMurray JJ, Adamopoulos S, Anker SD et al; ESC Committee for Practice Guidelines (2012) ESC Guidelines for the diagnosis and treatment of acute and chronic heart failure 2012: The Task Force for the Diagnosis and Treatment of Acute and Chronic Heart Failure 2012 of the European Society of Cardiology. Developed in collaboration with the Heart Failure Association (HFA) of the ESC. Eur Heart J 33(14): 1787-847.

Makaya M, Matsuoka S, Kato N (2013) Combination of depression and poor knowledge regarding heart failure: a strong determinant of selfcare behavior in patients with heart failure. Eur Heart J 34(1): 952.

National Institute for Health and Care Excellence (2010) Chronic heart failure: Management of chronic heart failure in adults in primary and secondary care. [CG5]. NICE, London. https://www.nice.org.uk/ guidance/cg5 (accessed 21 January 2016)

Rector TS, Kubo SH, Cohn JN (1987) Patients' self-assessment of their congestive heart failure. Part 2: Content, reliability and validity of a new measure, the Minnesota Living with Heart Failure questionnaire. Heart Failure Oct/Nov: 198-209

Riegel B, Lee CS, Dickson VV, Carlson B (2009) An update on the selfcare of heart failure index. J Cardiovasc Nurs 24(6): 485-97.

Rogers A, Addington-Hall JM, McCoy A et al (2002) A qualitative study of chronic heart failure patients' understanding of their symptoms and drug therapy. Eur J Heart Fail 4(3): 283-7

Salyer J, Schubert CM, Chiaranai C (2012) Supportive relationships, self-care confidence, and heart failure self-care. J Cardiovasc Nurs 27(5): 384-93.

Scottish Intercollegiate Guidelines Network (2007) Management of chronic heart failure: A national clinical guideline. SIGN, Edinburgh. http://www.sign.ac.uk/pdf/sign95.pdf (accessed 21 January 2016)

Shao JH, Chang AM, Edwards H, Shyu YI, Chen SH (2013) A randomized controlled trial of selfmanagement programme improves health-related outcomes of older people with heart failure. J Adv Nurs 69(11): 2458-69.

Smeulders ES, van Haastregt JC, Ambergen T, Janssen-Boyne JJ, van Eijk JT, Kempen GI (2009) The impact of a self-management group programme on health behaviour and healthcare utilization among congestive heart failure patients. Eur J Heart Fail 11(6): 609-16.

Smith $\mathrm{H}$, Hankins $\mathrm{M}$, Hodson A, George C (2009) Measuring the adherence to medication of elderly patients with heart failure: is there a gold standard? Int J Cardiol 145(1): 122-3.

Sutherland K (2010) Bridging the quality gap: heart failure. The Health Foundation, London 
Table 1:

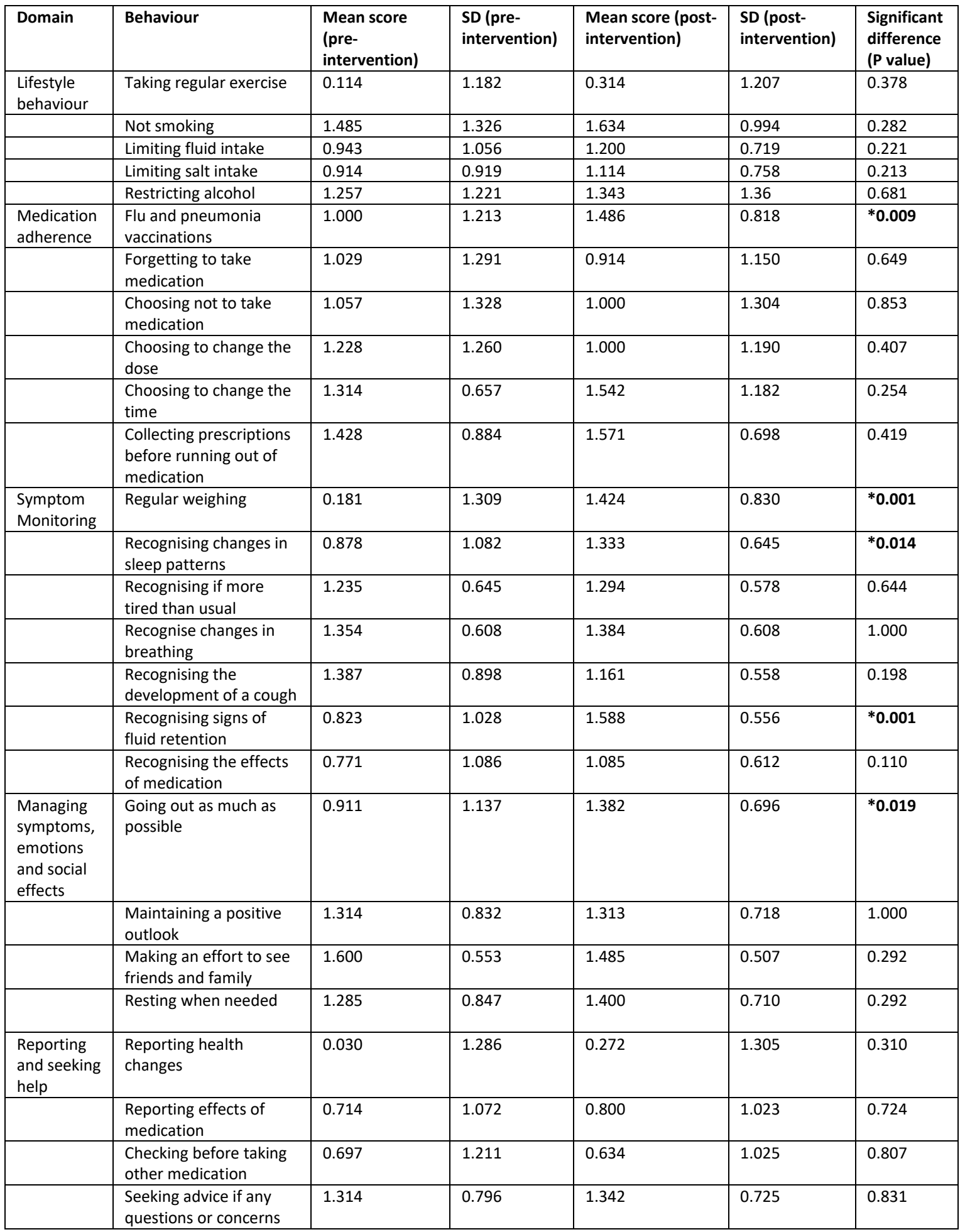

